\title{
MODELS AND METHODS FOR LOGISTICS HUB LOCATION: A REVIEW TOWARDS TRANSPORTATION NETWORKS DESIGN
}

\section{Carolina Luisa dos Santos Vieira* and Mônica Maria Mendes Luna}

Received January 21, 2016 / Accepted July 18, 2016

\begin{abstract}
Logistics hubs affect the distribution patterns in transportation networks since they are flowconcentrating structures. Indeed, the efficient moving of goods throughout supply chains depends on the design of such networks. This paper presents a literature review on the logistics hub location problem, providing an outline of modeling approaches, solving techniques, and their applicability to such context. Two categories of models were identified. While multi-criteria models may seem best suited to find optimal locations, they do not allow an assessment of the impact of new hubs on goods flow and on the transportation network. On the other hand, single-criterion models, which provide location and flow allocation information, adopt network simplifications that hinder an accurate representation of the relationship between origins, destinations, and hubs. In view of these limitations we propose future research directions for addressing real challenges of logistics hubs location regarding transportation networks design.
\end{abstract}

Keywords: logistics hub, location, literature review, transportation network.

\section{INTRODUCTION}

Logistics hubs are large-scale structures within which different logistics service providers collaborate in order to offer value-added services by sharing assets. Such hubs impact on the efficiency of transportation systems, since they directly affect the flow of goods. In order to achieve an increased efficiency, it is necessary to correctly position these hubs on a network. According to Li, Liu \& Chen (2011), the purpose of adequate location of a logistics hub is to make products available to different markets through the best possible connections, allowing for a better use of the logistics and transportation infrastructure available.

The process of locating a logistics hub tends to be somewhat more complex than for industrial facilities or distribution centers, since the hub is not intended to be used exclusively by one supply chain, but by a broader network of distribution. In these cases, hub-and-spoke topologies

*Corresponding author.

Departamento de Engenharia de Produção e Sistemas, Universidade Federal de Santa Catarina - UFSC, Florianópolis, SC, Brasil.

E-mails: carolina@nures.ufsc.br; monica.luna@ufsc.br 
are usually adopted, serving a wide variety of industries and products. Such configuration is common in the transportation of large volumes (Campbell \& O'Kelly, 2012; Lium, Crainic \& Wallace, 2009), where goods are concentrated in a few nodes, i.e. hubs, which act as connection points, instead of being sent directly from a supplier to their destinations (Ambrosino \& Sciomachen, 2012). This means that two major functions can be provided by hubs: i) consolidation/deconsolidation, and ii) switching, sorting or connecting (Campbell \& O'Kelly, 2012). Therefore, the decision on location should not be restricted by the definition of the number, site, and capacity of facilities (Simchi-Levi, Kaminsky \& Simchi-Levi, 2003), but must also take into account the allocation of products' flows and the network design itself (Campbell \& O’Kelly, 2012).

The location of logistics hubs is also considered to be a strategic and long-term decision, especially due to the large amount of capital invested and the length of time that facilities will be available. Already in 1994, Izquierdo (apud Dubke \& Pizzolato, 2011) pointed out that among the criteria which impact logistics hubs design, the location seemed to be a crucial decision element. The choice of site affects the success not only of operational activities itself (Tu et al., 2010), but also of supply chain management and of transportation network planning, ultimately influencing the distribution systems as a whole (Melo, Nickel \& Saldanha-da-Gama, 2009; Škrinjar, Rogić \& Stancović, 2012). Consequently, the design of a transportation network becomes also strategically important for businesses, as it impacts on how the goods will flow throughout the distribution channels available (Oktal \& Ozger, 2013).

As a result, the optimal location of a logistics hub may lead to reduced transportation costs, promote synchronization between production and consumption, ensure a balanced development of transportation systems, and achieve better overall benefits (Gao \& Dong, 2012; Lium, Crainic \& Wallace, 2009). A best location will effectively assist in the expansion of economies of scale, as well as increase competitive advantage, achieving higher customer satisfaction through more efficient transportation (Ding, 2013). Given the importance of these issues, this paper analyzes the existing literature on location of logistics hubs, presenting an overview of the modelling approaches taken, the solution techniques implemented, and their applicability to the context of such structures. Differently from other reviews on hub location, here we bring together the developments regarding the logistics hub framework, instead of focusing on the development of a particular model or solution approach.

This paper is organized as follows. Section 2 presents the review papers that have dealt with hub location so far. Section 3 describes the methodological procedures and some particularities encountered while performing this research. Context and perspectives of logistics hub location are provided in Section 4. The types of models used, general aspects of their formulation, and their corresponding solution methods are displayed in Section 5. Section 6 discusses the applicability of models and solution techniques in the context of the logistics hubs, especially regarding their role as part of transportation networks. Finally, Section 7 comprises the final considerations, including future research directions. 


\section{LITERATURE REVIEW}

Hub location is a well-established research field in operational research. This is ratified not only by the existence of journals dedicated to location science itself, but also by several review papers offering an overview of research progress over time. Although developments in the hub location area are mainly connected to the need to move people or products, this kind of problem formulation is also adopted in telecommunications, where data is distributed via hubs throughout information networks (Alumur \& Kara, 2008). In fact, one of the first reviews on hub location was dedicated to the context of communication network architecture, by Klincewicz (1998).

Yet, it was only after a period of ten years that Alumur \& Kara (2008) presented a new survey, fairly comprehensive, reviewing more than 100 articles related to hub location in general. The authors described mathematical models, solution techniques adopted, and benefits arising from the choice of one technique or another. They also identified the classic data sets available for the models' evaluation. A special section was dedicated on issues related to economies of scale, and how to include this feature on the models. The survey presented by Alumur \& Kara (2008) indicate that, since 2000, the focus of works has shifted from the definition and formulation of new problems to the investigation of new solution methodologies. In general, time and cost were the main criteria to be minimized, especially in freight transportation. The authors also pointed out the need to address multiple criteria decisions, especially with conflicting objectives, as well as to represent the transportation networks more adequately.

Aiming to extend the research of Alumur \& Kara (2008), Farahani et al. (2013) reviewed the literature on hub location from 2007 onwards. Besides presenting discrete problems, which were emphasized by Alumur \& Kara (2008), Farahani et al. (2013) also included continuous approaches. The classification of the literature followed the proposition of Alumur \& Kara (2008), although now with larger subdivisions to accommodate further modeling features, such as capacity limitation, multiple objectives, and network coverage. To Farahani et al. (2013), the consideration of multiple criteria and real world aspects are issues that still require improvement. Current logistics matters related to risk, sustainability, environmental impact, and globalization of supply chains are becoming increasingly more important in decision making. Furthermore, the influence of a hub on products' flows and the effects of traffic on a network also lack investigation.

Another work that surveys the literature on hub location was presented by Campbell \& O'Kelly (2012). Here the authors took a slightly different approach, evaluating the origins of the hub location problem (HLP), its evolution over time, and how it presents itself nowadays. The current state of the art is discussed with respect to large-scale problems, network topology, integration between costs and services, dynamic modelling, competition situations, stochasticity, and reliability. Campbell \& O'Kelly (2012) also described the relationship between the location of hubs and network design, which adds some special challenges to problem modelling and solving. Future research is in line with that indicated by Alumur \& Kara (2008) and Farahani et al. (2013). In particular, Campbell and O'Kelly (2012) pointed out that the models available are still limited in representing real transportation networks, and do not emphasize results related to spatial organization and allocation of flows throughout the arcs of the network. 
Although the majority of models available in the literature consider just one decision criteria, multiple-criteria decision making (MCDM) models have been increasingly adopted, allowing for a better representation of location issues. In light of this, Farahani, Seifi \& Asgari (2010) compiled a set of papers on the application of MCDM for facilities location in general. In these cases, in addition to classic criteria such as cost or coverage, at least one other criterion was considered, generally a conflicting one, such as environmental risk or service level. Works were classified according to the number of objectives and attributes considered, and solution methods were described. Future research directions highlighted the need to consider aspects related to reliability against flow disruption, data uncertainty, sustainability, and network design.

Finally, Melo, Nickel \& Saldanha-da-Gama (2009) reviewed the literature from the perspective of an application context: supply chain management. Here, facilities include not only hubs, but also industrial plants. The authors pointed out the criteria that should be taken into account when locating facilities within the scope of supply chain planning, as well as solution techniques adopted and some applications. Network structure, financial issues, risk management, and the incorporation of reverse logistics were also among the issues discussed in this paper. In addition to typical location and allocation decisions, the models presented evaluated capacity, inventory levels, procurement, production activities, vehicle routing, and/or modes of transportation. Still, the networks analyzed are considerably simplified, especially regarding the number of chain levels represented and the diversification of products handled. Regarding future research directions, Melo, Nickel \& Saldanha-da-Gama (2009) highlighted the need to improve the orientation of the models, since they mainly focus on economic factors, as well as to take into account uncertainties inherent in the supply chain scenario. The integration between operational and tactical and strategic decisions also requires further elaboration, as do reverse logistics activities.

In general, the review papers above survey the literature with regard to a specific type of problem, such as the HLP, or a modelling approach, such as MCDM. However, this segmentation makes it difficult to identify the available (or more adequate) approaches to deal with a specific situation, such as logistics hubs; in fact, the applicability of location models is a matter at constant debate. We found the work of Melo, Nickel \& Saldanha-da-Gama (2009) to go in this direction, extending the knowledge of a model's suitability for the supply chain management perspective, and allowing for better problem solving.

\section{RESEARCH METHODOLOGY}

For this survey, we adopted a content analysis approach, which aims for a systematic, quantitative, and qualitative description of the selected literature. Two databases were used in this research: Scopus and Emerald Insight. There, we initially identified the works that dealt with logistics hubs in general, instead of searching directly for facility location. This allowed us to limit the universe of papers to the logistics hub area, because the literature on location is quite extensive. Next, we searched for the papers that dealt with location, regardless of the approach adopted. Referenced papers or relevant literature reviews were added to complement the pool of articles. 
One major obstacle faced during this process relates to which terms should be used to define a logistics hub. Since there is no stated consensus in the literature on the logistics hub terminology, and since this research aimed to identify the models and methods that could be used to locate logistics hubs, we have considered a broader array of expressions: logistics hub, logistics center, freight village, or logistics platform. Among these, logistics center seems to be the one mostly used by authors.

A total of 20 papers were selected for analysis, in which we sought to identify: overall characteristics, such as context and modelling perspective; modes of transportation, connections and infrastructure available; objectives and criteria used for decision making; solution techniques and/or algorithms adopted; and, suggestions for future research. The content analysis of the above allowed us to observe and evaluate the properties and goals of the location models available, the situations where they were implemented, their applicability and limitations to the context of logistics hubs.

\section{CONTEXT, PERSPECTIVES, AND NETWORKS}

Although facility location problems have been studied in the early 20th century (Škrinjar, Rogić $\&$ Stancović, 2012), it was not until the 1950s that more elaborate approaches started to emerge for the location of interconnection points (Campbell \& O'Kelly, 2012). Goldman (1969) can be regarded as one of the first authors who modelled the transportation hub location problem. His paper pointed postal operations as an important application; in fact, the advent of express delivery firms in the late 1970s has also proved to be a practical motivation for further developments in this area (Campbell \& O'Kelly, 2012). Nevertheless, it was the seminal work of O'Kelly (1986) that set off the HLP as a new research agenda, which took into account the spatial interactions between location and transportation decisions (Kara \& Taner, 2011). O'Kelly studied the interactions between hubs for the United States inter-city air passengers' streams; it was the starting point for a large spectrum of applications based on the concept that location had an effect on the design of its associated networks (Kara \& Taner, 2011). These developments were further supported by the application of mathematical programming and heuristics techniques, and the increase in computational power. Subsequently, the need to consider a greater variety of decision criteria, both quantitative and qualitative, led to the consideration of other fields of investigation, such as MCDM, and formulations based on fuzzy logic.

Yet, despite the research interest in hub location, not many authors have applied their proposed approaches to solving problems in practice. The majority of works still evaluate applications with numerical data. This might be related, however, to the fact that their focus lies on the development or improvement of a given solution technique. On the other hand, the employment of primary data arise in two main situations: i) when new criteria are included in a model, in order to represent particular aspects of the problem, such as seen in Tu et al. (2010), Gao \& Dong (2012), Oktal \& Ozger (2013) and Klapita \& Švecová (2006); and ii) for the analysis of results and implications of facility location for a supply chain or the transportation network, 
e.g. Rahimi, Asef-Vaziri \& Harrison (2008), Ambrosino \& Sciomachen (2012), Lee, Huang \& Teng (2009), Dubke \& Pizzolato (2011) and Alumur, Kara \& Karasan (2012).

\subsection{Location perspectives}

Finding the best location for a logistics hub can be done using two different perspectives, which are related to the scope of the problem to be solved and the results obtained. While the first perspective is associated with a business or supply chain point of view, the second is broader and strives to improve the freight network and foster a better use of infrastructure through the planning of transportation systems. Both perspectives can be found in the literature as depicted in Table 1.

Table 1 - Perspectives of benefits obtained from the location of logistics hubs.

\begin{tabular}{|c|l|}
\hline Perspectives & Authors \\
\hline Business and supply chain & (Xiao \& Zhang, 2009), (Zhi \& Li, 2012), (Tu et al., 2010), \\
& (Ren et al., 2010), (Oktal \& Ozger, 2013), (Dubke \& Pizzo- \\
& lato, 2011), (Škrinjar, Rogić \& Stancović, 2012), (Feng, Li \& \\
& Zhang, 2013), (Zhi et al., 2010), (Klapita \& Švecová, 2006), \\
& (Alumur, Kara \& Karasan, 2012), (Wang \& He, 2009), (Li, Liu \\
& \& Chen, 2011), (Liu, Guo \& Zhao, 2012), (Kampf, Pruša \& \\
& Savage, 2011) \\
\hline Transport systems planning & (Turskis \& Zavadskas, 2010), (Rahimi, Asef-Vaziri \& Harrison, \\
& 2008), (Ambrosino \& Sciomachen, 2012) \\
\hline
\end{tabular}

In papers focused on the first perspective, the choice of location has a direct impact on economic aspects related to the implementation and fulfillment of operational activities within a supply chain (Wang \& He, 2009). In such cases, reductions in transportation costs and lead-time or increase in revenues are issues that prevail in decision making; i.e. the authors adopt a microeconomic point of view. According to (Škrinjar, Rogić \& Stancović, 2012), these benefits usually result from a better use of vehicle space and/or exploitation of transport capacities. This perspective is widely adopted in the literature and was found in 15 of the 20 papers analyzed.

On the other hand, the works of Rahimi, Asef-Vaziri \& Harrison (2008), Turskis \& Zavadskas (2010) and Ambrosino \& Sciomachen (2012) address location from a transport system perspective, dealing with infrastructure planning on a regional level. For these authors, a transport system that includes logistics hubs could contribute to the establishment of a network that allows for a region to compete economically and efficiently in both local and regional markets. Some papers show a predominantly public goal, where there is a concern with obtaining benefits for the society. Maximizing the support provided by the transport systems in this perspective would also imply on the reduction of adverse factors, mainly caused by an inadequate use of the network, which results in damage to the environment and public health, such as air and noise pollution (Ambrosino \& Sciomachen, 2012; Rahimi, Asef-Vaziri \& Harrison, 2008). 
While the focus of research is the location and construction of new facilities, the type of investment required for the implementation of hubs is generally not discussed. Although construction costs are taken into consideration when there is a choice between different location sites, they seem to be related only to the new facilities, and not to the network design. Kampf, Pruša \& Savage (2011) and Zhi \& Li (2012) claim that there should be a public-private partnership when locating logistics hubs, ensuring both the provision of value-added services and a positive outcome for society. In these cases, public funding should be directed to the construction or maintenance of infrastructure, while private capital would be better employed for construction of facilities, acquisition of equipment, and implementation of information and communication technologies (Kampf, Prů̌̌a \& Savage, 2011).

\subsection{Transportation networks considered}

The flows of goods that could be handled in a logistics hub depend directly on the distribution channels and connections available in the network. The surveyed papers consider a variety of transportation modes, as outlined in Table 2. There is a predominance of works dealing with road transport, followed by analyses that include hubs connected to railways. Airways, considered only by five authors, are always integrated with a multimodal platform. Given that multimodality is considered by many authors to be a basic feature of a logistics hub, it is understandable that the majority of papers that discuss the network consider more than one mode of transportation. On the other hand, there are several works, e.g. Wang \& He (2009), Turskis \& Zavadskas (2010), Li, Liu \& Chen (2011), Liu, Guo \& Zhao (2012), Zhi \& Li (2012) and Škrinjar, Rogić \& Stancović (2012), that do not mention the transportation mode used.

Table 2 - Transportation modes available in networks.

\begin{tabular}{|l|c|c|c|c|c|c|}
\hline \multicolumn{1}{|c|}{ Author } & Roadway & Railway & Maritime & Waterway & Airway & Other aspects \\
\hline (Xiao \& Zhang, 2009) & $\checkmark$ & & & & & \\
(Tu et al., 2010) & & & & & $\checkmark$ & \\
(Gao \& Dong, 2012) & $\checkmark$ & $\checkmark$ & & $\checkmark$ & & $\checkmark$ \\
(Rahimi, Asef-Vaziri \& Harrison, 2008) & $\checkmark$ & $\checkmark$ & & & & $\checkmark$ \\
(Ren et al., 2010) & $\checkmark$ & & & & & $\checkmark$ \\
(Oktal \& Ozger, 2013) & & & & & \\
(Ambrosino \& Sciomachen, 2012) & $\checkmark$ & $\checkmark$ & & & \\
(Lee, Huang \& Teng, 2009) & $\checkmark$ & & $\checkmark$ & & \\
(Dubke \& Pizzolato, 2011) & $\checkmark$ & $\checkmark$ & & & & $\checkmark$ \\
(Feng, Li \& Zhang, 2013) & $\checkmark$ & $\checkmark$ & & & $\checkmark$ \\
(Klapita \& Švecová, 2006) & $\checkmark$ & & & & \\
(Alumur, Kara \& Karasan, 2012) & $\checkmark$ & & & & $\checkmark$ & $\checkmark$ \\
(Kampf, Prơša \& Savage, 2011) & $\checkmark$ & $\checkmark$ & & $\checkmark$ & $\checkmark$ & $\checkmark$ \\
\hline
\end{tabular}

Liu, Guo \& Zhao (2012) point out that a location model should always consider the transportation network as a system. Hence, the evaluation of location alternatives should also take into account possible connections and the accessibility to the transportation network (Ambrosino \& 
Sciomachen, 2012). Railway, waterway and roadway links, which enable multimodal connections at transshipment points are regarded by Kampf, Prů̌̌ \& Savage (2011), Ambrosino \& Sciomachen (2012), Gao \& Dong (2012) and Feng, Li \& Zhang (2013).

The infrastructure's conservation state and the use of its capacity are issues that impact the performance of a transport system. According to Zhi \& Li (2012), these aspects are directly related to the service level achieved when using the infrastructure, which in turn directly affects the services offered in a logistics hub. The assessment of the infrastructure conditions further indicates whether or not it is possible to remodel existing facilities to be used as hubs, which could save money and time (Feng, Li \& Zhang, 2013; Ren et al., 2010). On the other hand, observing traffic conditions and flow patterns not only assists in the identification of potential sites for a logistics hub, but aids in evaluating the flow changes and their environmental impacts due to more intense traffic or congestion (Liu, Guo \& Zhao, 2012; Ren et al., 2010).

\section{MODELS AND SOLUTION TECHNIQUES FOR THE LOCATION OF LOGISTICS HUBS}

The location models available in the surveyed literature were sorted into two categories, based on the number of decision criteria considered: multi-criteria or single-criterion. This classification is related not only to the model itself and type of results obtained, but also to the solution techniques adopted.

Different aspects can be considered during modeling, which are used either as decision criteria or model restrictions. They comprise: i) transport, related to transportation costs, time or distance travelled; ii) hub functionality, regarding the activities carried out on the hub, capacity, skilled labor availability, operating costs, fees, etc.; iii) investment, concerning the required amount of capital for the construction of facilities; iv) supply and demand, which deals with the availability of products and volumes to be handled, including traffic; v) market, considering the proximity to customers and potential for coverage expansion; vi) policy, which includes the development of policies, current legislation, and benefits of tax incentives; and vii) environment, linked to terrain characteristics, geography, and environmental protection. Table 3 presents the types of models and features taken into account, per author.

Meanwhile, regardless of model's features, it is a consensus among authors that the decision on location begins with the pre-selection of a set of potential sites where the hub could be implemented, particularly if the network considered has a large number of possible locations (Zhi \& $\mathrm{Li}, 2012$ ). Indeed, to test all possible combinations becomes impracticable. Although most authors do not make clear how this pre-selection is made, several criteria could be identified which are related to product flow, supply and demand of products, and available infrastructure.

According to Gao \& Dong (2012) and Dubke \& Pizzolato (2011), logistics hubs should be located at the intersection of large streams of flow, or very close to major transport links, especially in order to take advantage of multimodality. In addition to ensuring the existence of a greater volume of cargo that could be handled in the hub, this would encourage a better use of the 
Table 3 - Types of models and aspects taken into account.

\begin{tabular}{|l|c|c|c|c|c|c|c|}
\hline \multicolumn{2}{|c|}{ Authors } & \multicolumn{9}{c|}{ Aspects } \\
\hline & Transp. & Function. & S/D* & Invest. & Market & Policy & Environm. \\
\hline (Lee, Huang \& Teng, 2009) & $\checkmark$ & $\checkmark$ & $\checkmark$ & $\checkmark$ & $\checkmark$ & $\checkmark$ & \\
(Tu et al., 2010) & $\checkmark$ & $\checkmark$ & $\checkmark$ & $\checkmark$ & $\checkmark$ & $\checkmark$ & $\checkmark$ \\
(Ren et al., 2010) & $\checkmark$ & $\checkmark$ & & $\checkmark$ & $\checkmark$ & $\checkmark$ & $\checkmark$ \\
(Turskis \& Zavadskas, 2010) & & & & $\checkmark$ & $\checkmark$ & & \\
(Li, Liu \& Chen, 2011) & & & $\checkmark$ & $\checkmark$ & & & $\checkmark$ \\
(Kampf, Pruša \& Savage, 2011) & $\checkmark$ & & & $\checkmark$ & & & \\
(Feng, Li \& Zhang, 2013) & $\checkmark$ & $\checkmark$ & $\checkmark$ & $\checkmark$ & & & \\
(Klapita \& Švecová, 2006) & $\checkmark$ & $\checkmark$ & $\checkmark$ & & & & \\
(Rahimi, Asef-Vaziri \& Harrison, 2008) & $\checkmark$ & $\checkmark$ & $\checkmark$ & $\checkmark$ & & & \\
(Wang \& He, 2009) & $\checkmark$ & $\checkmark$ & $\checkmark$ & $\checkmark$ & & & \\
(Xiao \& Zhang, 2009) & $\checkmark$ & $\checkmark$ & $\checkmark$ & $\checkmark$ & & & \\
(Zhi et al., 2010) & $\checkmark$ & & $\checkmark$ & $\checkmark$ & & & \\
(Dubke \& Pizzolato, 2011) & $\checkmark$ & $\checkmark$ & $\checkmark$ & & & & \\
(Zhi \& Li, 2012) & $\checkmark$ & $\checkmark$ & $\checkmark$ & & & & \\
(Gao \& Dong, 2012) & $\checkmark$ & & & & & & $\checkmark$ \\
(Ambrosino \& Sciomachen, 2012) & $\checkmark$ & $\checkmark$ & $\checkmark$ & & & & \\
(Škrinjar, Rogić \& Stancović, 2012) & $\checkmark$ & $\checkmark$ & $\checkmark$ & $\checkmark$ & $\checkmark$ & & $\checkmark$ \\
(Alumur, Kara \& Karasan, 2012) & $\checkmark$ & $\checkmark$ & $\checkmark$ & & & & \\
(Liu, Guo \& Zhao, 2012) & $\checkmark$ & & $\checkmark$ & & & & \\
(Oktal \& Ozger, 2013) & $\checkmark$ & $\checkmark$ & $\checkmark$ & & & & \\
\hline
\end{tabular}

*supply and demand.

existing infrastructure. Rahimi, Asef-Vaziri \& Harrison (2008), for example, rated potential location sites based on traffic distribution and total distances travelled by vehicles. On the other hand, Ambrosino \& Sciomachen (2012) considered the possibilities of exchange between modes as a basis for the pre-selection.

Defining the initial set of potential location sites could also be based on criteria related to the location of supply and demand of goods. Boudouin \& Luna (2012) suggest that areas where product consumption is concentrated could be used as foundation to identify the need for a logistics hub, especially when urban transportation is at stake. Ambrosino \& Sciomachen (2012) go in the same direction, adopting a procedure that considers government data on supply and demand of products. Alumur, Kara \& Karasan (2012), in turn, pre-select sites based on population and industrialization of cities and regions.

Identifying the existing infrastructure that could accommodate a logistics hub is the third criterion adopted in pre-selection. Tu et al. (2010) and Oktal \& Ozger (2013) discuss the possibilities of installing hubs in airports that already have feasible features, such as size of landing runway and capacity to receive a greater number of aircrafts resulting from an increased volume of airflow. In line with this idea, Feng, Li \& Zhang (2013) consider existing railroad stations and select those best suited to support a hub. The authors evaluate not only the physical conditions of the railroad station, but also the regional support, the traffic geography and environmental 
development. On the other hand, Lee, Huang \& Teng (2009) verify the storage conditions of existing distribution centers that could develop into transshipment facilities for maritime shipping, as well as the distance between the hub and ports in the same region.

\subsection{Multi-criteria models}

The location of logistics hubs is a complex problem, in which decision is affect by the context, the availability of information, and the importance given to the evaluation criteria (Lee, Huang $\&$ Teng, 2009). Therefore, according to these authors, decision should be made based on multiple criteria, supported by quantitative and qualitative data. Multi-criteria models typically allow conflicting criteria to be taken into account, which would then be evaluated by decision makers in order to establish preferences among possible location sites. Among the papers surveyed, the ones that take in account the greater amount of criteria area proposed by Lee, Huang \& Teng (2009), Ren et al. (2010) and Tu et al. (2010).

Formulating a multi-criteria model usually starts by identifying the most relevant decision criteria. Here the aspects described in Table 3 could be directly used as decision criteria. Next, the pre-selected sites would have their performance evaluated according to each criterion. The way in which the evaluation is carried out depends on the solving technique adopted, which can result in one optimal solution or a set of good alternatives. In this case, results could also be evaluated and ranked by means of sensitivity analysis.

Quantitative parameters are the most used, probably due to the ease of obtaining data and related information. Within this scope, all authors seem to agree that the investment required for construction should be considered in the models, as well as costs related to transportation activities. Functional aspects are less frequently used, such as issues related to product handling, and supply/demand information.

Qualitative criteria, on the other hand, require more complex analysis and are mainly grounded on expert knowledge. Nonetheless, the possibility of evaluating this type of criteria is highlighted as the major advantage of multi-criteria modeling. Therefore, they are found in larger quantities and practically in all modes in this category, except for Kampf, Prǔ̌a \& Savage (2011). The potential for facilities expansion, availability of skilled labor and proximity to marked are considered by Lee, Huang \& Teng (2009), Ren et al. (2010), Tu et al. (2010) and Turskis \& Zavadskas (2010), as well as the availability of support services, such as energy provision and waste management. Issues related to regional development policies, legislation, and tax incentives are taken into account by Lee, Huang \& Teng (2009), Ren et al. (2010) and Tu et al. (2010). Lee, Huang \& Teng (2009), Tu et al. (2010) and Li, Liu \& Chen (2011) also point out the need to consider geographic, topographic, and hydrological aspects of the available land for the hub installation. Finally, environmental factors related to noise pollution and environment degradation are evaluated by Ren et al. (2010) and Li, Liu \& Chen (2011).

More than selecting the best hub location, multi-criteria models expose some other interesting results. Turskis \& Zavadskas (2010) show that the participation of stakeholders is crucial in the 
modelling process, since they allow for the assessment of qualitative criteria, such as expansion possibilities and market proximity. Lee, Huang \& Teng (2009), in turn, give examples of strategies for the development of logistics hubs, pointing out the importance of cooperation between business and the public sector in strengthening the competitiveness of a region. Both sets of authors adopt a macroeconomic perspective, focusing on infrastructure planning.

\subsection{Solution techniques for multi-criteria models}

Multi-criteria models are usually solved by a specific set of tools, characteristic of MCDM. The combination of more than one solution technique seems common in the papers surveyed. Among the methods found, the most adopted ones are fuzzy sets and the analytic hierarchy process (AHP), followed by weighted sum, goal programming and technique for order of preference by similarity to ideal solution (TOPSIS). Other techniques, e.g. heuristics, are seldom applied, as can be seen in Table 4 .

Table 4 - Solution techniques adopted for solving multi-criteria models.

\begin{tabular}{|l|c|c|c|c|c|c|c|c|c|}
\hline \multirow{2}{*}{ Authors } & \multicolumn{3}{c|}{ Typical MCDM } & \multicolumn{3}{c|}{ Others } \\
\hline & AHP* & $\begin{array}{c}\text { Fuzzy } \\
\text { sets }\end{array}$ & $\begin{array}{c}\text { Weighted } \\
\text { sum }\end{array}$ & $\begin{array}{c}\text { Goal } \\
\text { programming }\end{array}$ & TOPSIS** & SWOT*** & $\begin{array}{c}\text { Genetic } \\
\text { algorithm }\end{array}$ & $\begin{array}{c}\text { Tabu } \\
\text { search }\end{array}$ & $\begin{array}{c}\text { Simulated } \\
\text { annealing }\end{array}$ \\
\hline (Lee, Huang \& Teng, 2009) & $\checkmark$ & $\checkmark$ & & & & $\checkmark$ & & & \\
(Tu et al., 2010) & $\checkmark$ & & & $\checkmark$ & & & & & \\
(Ren et al., 2010) & & $\checkmark$ & & & & & & & \\
(Turskis \& Zavadskas, 2010) & $\checkmark$ & $\checkmark$ & & & & & & \\
(Li, Liu \& Chen, 2011) & & $\checkmark$ & & & & & & \\
(Kampf, Pruša \& Savage, 2011) & $\checkmark$ & & $\checkmark$ & & & & & $\checkmark$ \\
(Feng, Li \& Zhang, 2013) & & & & & & & & $\checkmark$ \\
\hline
\end{tabular}

*analytical hierarchy process; **technique for order of preference by similarity to ideal solution;

***strengths, weaknesses, opportunities and threats.

Due to the possibility of incorporating qualitative elements and uncertainty into the decision variables, Klapita \& Švecová (2006) and Li, Liu \& Chen (2011) indicate the fuzzy sets formulation as one of the most suitable tools for solving multi-criteria models. This approach allows decision makers to use inaccurate or incomplete data to find a solution (Turskis \& Zavadskas, 2010), without giving up the quantitative parameters. While the qualitative parameters are depicted in terms of fuzzy values, with the help of linguistic variables for their evaluation, the quantitative ones can be represented directly by numerical values and/or statistics. Furthermore, uncertainty can be represented by probability distributions (Ding, 2013). Although the results obtained with this method are concrete outcomes, its credibility depends intrinsically on the skills of decision makers and their ability and experience in selecting the most appropriate level of preference when comparing decision criteria (Klapita \& Švecová, 2006).

AHP is also a method that allows one or more decision makers to express their preferences by either numeric values or linguistic variables (Kampf, Prǔš \& Savage, 2011). However, it appears to never be used alone, but in combination with another technique. When dealing with multiple 
criteria, the AHP is commonly applied as a first step of decision making, being employed to classify criteria in a scale of importance, such as done by Lee, Huang \& Teng (2009), Tu et al. (2010), Turskis \& Zavadskas (2010) and Kampf, Prǔ̌a \& Savage (2011).

Still in the MCDM field, we found the adoption of a weighted sums approach by Kampf, Pruša \& Savage (2011). One of the simplest methods of multi-criteria evaluation, it is applied only when all data can be expressed in the same unit, which makes its adoption quite limited. On the other hand, Tu et al. (2010) chose goal programming, which allows for detailed information to be incorporated into the problem's structure, aiding in the determination of the requirements that would maximize the customers' satisfaction based on limited resources. Finally, Li, Liu \& Chen (2011) used the TOPSIS method, combined with axiomatic fuzzy sets in the initial stage of the decision process rather than with AHP.

Other non-traditional methods of multi-criteria decision making were also identified. Focused on the development of logistics hubs and transportation networks, Lee, Huang \& Teng (2009) applied a SWOT matrix to evaluate the competitiveness of a number of possible sites for the hub installation. Feng, Li \& Zhang (2013), in turn, propose a heuristic method which combines genetic algorithm, tabu search, and simulated annealing in order to minimize construction costs and customer costs.

\subsection{Single-criterion models}

Although real world logistics hub location problems have a multi-criteria nature, they are often reduced to simplify their solution (Alumur, Kara \& Karasan, 2012, Škrinjar, Rogić \& Stancović, 2012). The literature shows that there is an emphasis on the use of single-criterion decision models, especially in recent years.

Single-criterion models adopt a similar formulation to the hub location problem (HLP) in almost all cases surveyed, except for Zhi \& Li (2012), Gao \& Dong (2012) and Liu, Guo \& Zhao (2012). This type of formulation deals with the location of facilities and the allocation of product flows between origins, hubs, and destinations, in order to distribute the goods through minimum cost paths Ambrosino \& Sciomachen (2012). In these models, the transportation network is usually represented by a graph, composed of origin, destination and hub nodes, arcs connecting hubs with origins and destinations, and arcs linking hubs among themselves in case more than one hub should be installed. Transshipment nodes are not included in HLP models. Also, although the original formulation of the HLP allowed direct connections between origins and destinations, the absence of such connections has become a basic feature of HLP models, as defined by Campbell in 1994 Campbell \& O'Kelly (2012); i.e. origins and destinations can only be connected via one or more hubs.

An important feature that differentiates HLP models is the type of flow allocation allowed. Here, two different concepts could be identified: single allocation, where each source and each destination is allocated to only one hub, as show in Klapita \& Švecová (2006), Zhi et al. (2010) and Škrinjar, Rogić \& Stancović (2012), and multiple allocation, which allows non-hub nodes 
to be connected to more than one hub, as depicted by Rahimi, Asef-Vaziri \& Harrison (2008), Dubke \& Pizzolato (2011), Ambrosino \& Sciomachen (2012), Oktal \& Ozger (2013). Škrinjar, Rogić \& Stancović (2012) consider that multiple allocation provides the most complete allocation options, since they allow more flexibility in terms of connections available. In general, the benefits obtained with the location are inversely proportional to the amount of links required to connect the nodes in the network, and, consequently, to the transportation costs, which result from economies of scale achieved by a better network design (Campbell \& O'Kelly, 2012).

Flow allocation in a HLP model is linked to the adoption of a discount factor, with a value between 0 and 1, which indicates the range of economies of scale that can be achieved with the use of a hub. They are usually employed to lower the total transportation costs. This coefficient can be used in two different ways, depending on the connections available between the hubs. If the hubs to be opened are not connected, or if there is only one hub, the discount factor is applied to all arcs connected to that hub, leading to a reduction of the transportation costs on these arcs. If two or more hubs are connected, then the discount factor is applied to the inter-hub arcs (Campbell \& O'Kelly, 2012; Goldman, 1969; O'Kelly, 1986). In this case, Škrinjar, Rogić $\&$ Stancović (2012) point out that the transportation costs between the hubs end up being lower than those of other arcs, resulting from a better use of the infrastructure available.

Nonetheless, defining the discount factor would require a specific and long study that, to the best of our knowledge, has not yet been carried out. The value of the discount factor has usually been defined based on interviews (Alumur, Kara \& Karasan, 2012), or taken from the literature (Oktal \& Ozger, 2013). According to Campbell \& O'Kelly (2012), the discount factor could be related to the transportation mode used, ranging from 0.1 for rail to 1 for road. Although Kimms (2006) point out that this coefficient can be variable, depending on factors such as volume of flow in the arcs (Campbell \& O'Kelly, 2012), most authors still use a constant value due to the complexity that a variable factor could bring to the model. In order to work around this issue, Campbell \& O'Kelly (2012) observed an increase in the use of sensitivity analysis for evaluating the model's behavior with a wide range of discount factors.

A further proposal for logistics hub location models deals with the representation of the network through geographic coordinates of origins and consumption points (Liu, Guo \& Zhao, 2012). Zhi \& Li (2012), on the other hand, concentrate on the solution method and do not present a structure model for the problem.

As the network arcs are usually public roads, the addition of new arcs is not a concern in HLP models (Campbell \& O' Kelly, 2012). This, in fact, is a feature of a different category of models, called network design problems. However, when dealing with the location of more than one hub in the HLP, authors might consider new arc projects for inter-hub connections. Alumur, Kara \& Karasan (2012) take this aspect into account, assessing not only the sites and number of hubs to be opened, but also how they will be connected between each other and the transportation modes used for that. 
Single-criterion models seek to optimize different objective functions, often related to economic or financial matters, as shown in Table 5. Among these, the most common ones pursue costs minimization, either of transportation or total costs. Dubke \& Pizzolato (2011), on the other hand, aim at maximizing the revenue. Other goals might also be related to minimizing the travelled distances, which could be indirectly related to financial results, as well as service level and market coverage. This idea is adopted by Zhi \& Li (2012), who take a market perspective in order to reach the largest number of customers possible. In turn, Rahimi, Asef-Vaziri \& Harrison (2008) point out that not only economic issues should be evaluated, but also social costs. Although they are usually not embedded in the prices payed by hub users, social costs have shown an increased importance as a critical element in sustainable transportation systems.

Table 5 - Objective functions adopted in single-criterion models.

\begin{tabular}{|c|c|c|c|c|}
\hline \multirow[b]{2}{*}{ Authors } & \multicolumn{4}{|c|}{ Objective function } \\
\hline & $\begin{array}{l}\text { Min. } \\
\text { transport costs }\end{array}$ & $\begin{array}{l}\text { Min. } \\
\text { total costs }\end{array}$ & $\begin{array}{c}\text { Max. } \\
\text { revenue }\end{array}$ & $\begin{array}{c}\text { Max. } \\
\text { coverage }\end{array}$ \\
\hline $\begin{array}{l}\text { (Xiao \& Zhang, 2009) } \\
\text { (Zhi \& Li, 2012) } \\
\text { (Gao \& Dong, 2012) } \\
\text { (Rahimi, Asef-Vaziri \& Harrison, 2008) } \\
\text { (Oktal \& Ozger, 2013) } \\
\text { (Ambrosino \& Sciomachen, 2012) } \\
\text { (Dubke \& Pizzolato, 2011) } \\
\text { (Škrinjar, Rogić \& Stancović, 2012) } \\
\text { (Zhi et al., 2010) } \\
\text { (Klapita \& Švecová, 2006) } \\
\text { (Alumur, Kara \& Karasan, 2012) } \\
\text { (Wang \& He, 2009) } \\
\text { (Liu, Guo \& Zhao, 2012) }\end{array}$ & $\begin{array}{l}\checkmark \\
\checkmark \\
\checkmark \\
\checkmark \\
\checkmark\end{array}$ & $\begin{array}{l} \\
\checkmark \\
\checkmark\end{array}$ & $\checkmark$ & $\checkmark$ \\
\hline
\end{tabular}

Quantitative aspects are thus predominant in decision making with single-criterion models. Except for Gao \& Dong (2012), all models of this type consider data on transport and origin and destination of goods. Next, we found 15 of the 20 papers to adopt functional criteria. Data on the volume of investments is used in fewer cases, as can be seen in Table 3, and may be part of total cost minimizing object functions. Geographic characteristics of the terrain are also seldom applied.

Adding qualitative parameters is unusual in single-criterion models. Nonetheless, they could be found in the works of Gao \& Dong (2012) and Škrinjar, Rogić \& Stancović (2012). While the former believe that environmental protection issues are important, the latter add in the interaction of the logistics hubs with the market by evaluating the proximity between them.

Solving single-criterion models results not only in finding a hub location. The optimal amount of facilities required is obtained by Rahimi, Asef-Vaziri \& Harrison (2008), Xiao \& Zhang (2009), 
Alumur, Kara \& Karasan (2012) and Oktal \& Ozger (2013). In such cases, the pre-selected site set is tied to a restriction on the maximum number of hubs that could be installed. Results related to the allocation of origin and destination nodes are seldom found, highlighted only by Rahimi, Asef-Vaziri \& Harrison (2008) and Dubke \& Pizzolato (2011). In turn, the model proposed by Dubke \& Pizzolato (2011) goes deeper into the functionality matter, identifying the logistics services to be performed in each new hub.

\subsection{Solution techniques for single-criterion models}

There is a mixed set of solution methods for single-criterion models, ranging between heuristic, exact, and stochastic ones. But, unlike for multi-criteria, we did not find a preferred set of techniques for solving single-criterion models. Nonetheless, heuristic approaches seem to be more frequently used to solve HLP models. An overview of the techniques adopted is shown in Table 6.

After evaluating a variety of methods, Škrinjar, Rogić \& Stancović (2012) considered the genetic algorithm to be the most suitable for logistics hub single-criterion location, although they do not present an implementation. We observed the application of this method in two instances. Zhi et al. (2010) adopted particle swarm optimization, which combines both evolutionary features of genetic algorithms and probabilistic search of simulated annealing. Also Xiao \& Zhang (2009) worked with a combination of genetic algorithm, but in this case with an ant colony heuristic.

The ant colony heuristic by itself is adopted by Zhi \& Li (2012). Ambrosino \& Sciomachen (2012), in turn, combined traffic flow information obtained through a geographic information system (GIS) with a shortest path algorithm to find the best location in multimodal networks. Still in the field of heuristics, Alumur, Kara \& Karasan (2012) proposed their own technique, based on set covering, for solving a problem that combined network design and allocation in the same model. This was justified due to the complexity of the proposed problem, which was quite difficult to solve with the techniques available in the literature. According to the authors, the results were considered to be of good quality and to have been achieved in reasonable computing time.

Traditional exact techniques of deterministic optimization also find their place in solving logistics hub location problems. This is the case for mixed integer programming (MIP) and mixed integer linear programming (MILP), which were applied by Oktal \& Ozger (2013) and Dubke \& Pizzolato (2011), respectively. Liu et al. (2012), although highlighting a variety of issues that influence this kind of decision, ended up using the gravity center method, a less elaborate tool based on geographic coordinates. In order to evaluate aspects related to network flows, Rahimi, Asef-Vaziri \& Harrison (2008) and Gao \& Dong (2012) solved their problem through spatial analysis with the aid of GIS. On the other hand, Rahimi, Asef-Vaziri \& Harrison (2008) combined spatial analysis with partial weighted sum and with a procedure for constructing contour lines; however, when dealing with multiple hubs, the authors do not describe the method used. 


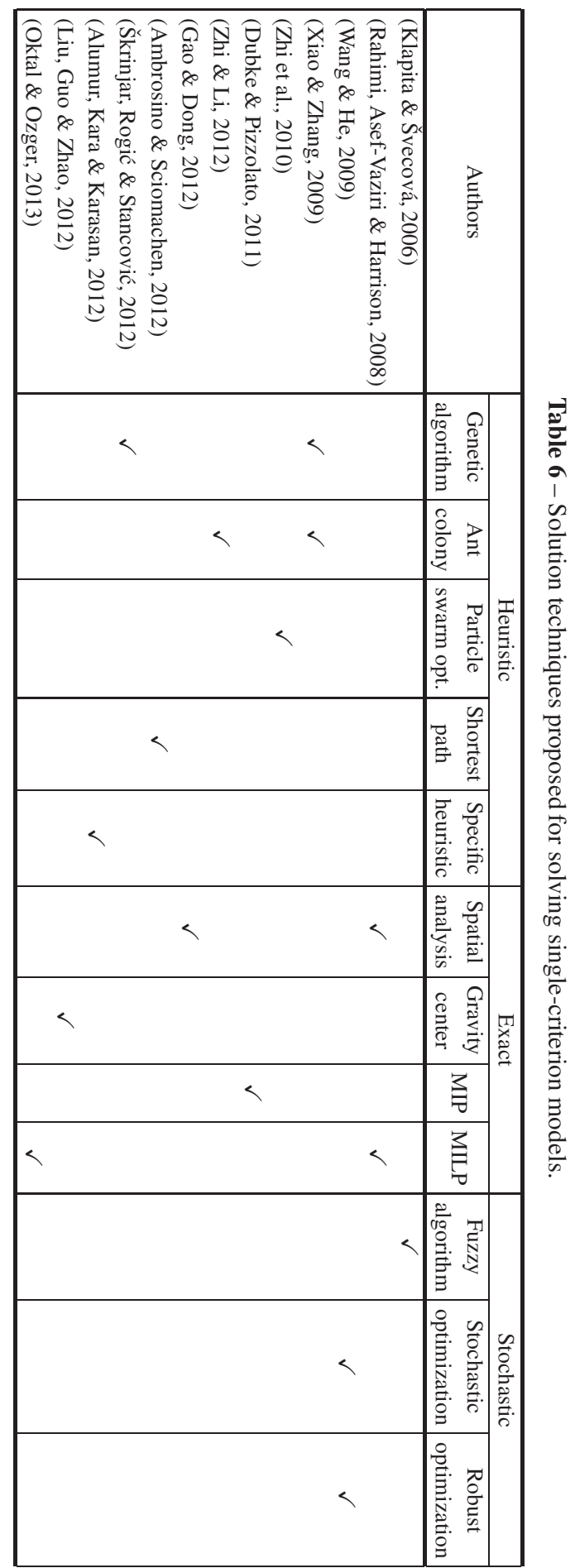

Pesquisa Operacional, Vol. 36(2), 2016 
Based on the premise that changes in input parameters may impact the decision on the number of hubs to be installed, their location, and flow allocation, Klapita \& Švecová (2006) and Wang $\&$ He (2009) claimed the adoption of measures to overcome uncertainty and variability to be necessary. Wang \& He (2009) investigated these aspects by considering demand uncertainty in a variety of economic scenarios, while other model parameters were kept deterministic. In this case, robust optimization was compared against stochastic optimization: according to the authors, the first allowed for a better representation of uncertainties while effectively reducing the risks in decision making when compared to the second. Klapita \& Švecová (2006) also performs comparisons between different solving tools that deal with the variability of parameters: sensibility analysis and fuzzy analysis. The authors propose an algorithm that employs principles of fuzzy logic, but does not depend on the skill of decision makers. According to them, the advantage of this proposal lies in identifying a best solution that, as pointed out by Wang \& He (2009), is resistant to future changes in the model.

Authors such as Dubke \& Pizzolato (2011) and Alumur, Kara \& Karasan (2012) also shed light on sensitivity analysis. They evaluate the model's outcomes regarding the number of hubs to be installed and their impact on the volume of products handled, facilities capacities, transportation costs, and revenue. Ambrosino \& Sciomachen (2012), also performs sensitivity analysis, but from a perspective of traffic reduction. Wang \& He (2009), in turn, evaluate the model's behavior according to different economic scenarios; however, they do so by using different solving techniques instead of sensitivity analysis. Lastly, Rahimi, Asef-Vaziri \& Harrison (2008) test the network sensitivity to the number of hubs that can be installed, evaluating the total travelled distances.

\section{APPLICABILITY OF MODELS AND SOLUTION TECHNIQUES FOR TRANSPORTATION NETWORKS}

In light of the concept of a logistics hub and its role in transportation networks, it is important to reflect on the adequacy and applicability of location models and solution techniques available to solve such problems in this context.

The type of model adopted seems to be directly related to the perspectives and goals of the papers surveyed. Models that seek to evaluate strategies, transportation network settings, or infrastructure planning and expansion, adopt predominantly a multi-criteria approach. This choice is mainly justified by the advantages of incorporating qualitative criteria, especially those related to policies, legal matters, and relationships with the market. They can be seen as models that encompass a macroeconomic view, which could be used to guide the improvement of a region's competitiveness. In turn, when the focus is on the benefits for those using the hub, it is evident that the choice is in favor of single-criterion models. They take a microeconomic view, evaluating aspects such as cost reduction and revenue increase. Although infrastructure investments, which depend mainly on the public sector, should be planned taking into consideration the goals of industries and logistics service providers and their customers, these two perspectives were not addressed together by one single model. 
If we consider the variety of qualitative and quantitative aspects that influence decision making when locating logistics hubs, then multi-criteria models seem to be more adequate. They have the advantage of being quite flexible, encompassing not only conflicting criteria, but also aggregating views of different stakeholders. However, they do not provide the means to evaluate flow distribution and its impact on the transportation network; at least, none of the models available in the surveyed literature brought results in this matter. Yet this kind of information would be of great importance when dealing with strategic decisions, especially regarding infrastructure planning.

Bearing this in mind, HLP models may seem more comprehensive, since they allow both hub location and flow allocation to be performed throughout a network. Although they have been seen, over time, to be broadly applicable to many network topologies, the models' abstract nature, apparent simplicity, and generality limit their ability to accurately represent important features of logistics systems (Campbell \& O'Kelly, 2012). Three main issues hinder the application of HLP models in large-scale networks which include logistics hubs: i) the absence of direct connections between pairs of origins and destinations; ii) the requirement that all products should flow straight through hubs, and at least through one hub; and iii) the simplification of paths and connections between origins, destinations, hubs, and other network nodes.

The lack of direct connections between origins and destinations in HLP models is the first issue that calls for our attention. This absence is justified by Campbell \& O'kelly (2012): the authors consider that such connections would be used only for large flows, especially full load trucks, which would naturally be transported straight from suppliers to customers. However, excluding these flows would prevent them from taking advantage of value added services that could be provided in the hub. This also implies that, for other flows, it would always be better to use routes that go through a hub. This does not correspond to reality, as it cuts out the use of other route options, which could end up being more profitable or guaranteeing a determined service level. This may even artificially overload the hub's usage, negatively impacting constructive aspects related to facilities' capacity and service dimensioning, leading ultimately to unnecessary investments.

Representing the routes through direct connections between origins and hubs, and between hubs and destinations makes it difficult to observe the real distribution of flows. This hinders the analysis from an infrastructure use perspective, as well as the evaluation of flow changes resulting from the implementation of a logistics hub. The use of HLP models itself actually leads us to believe that this may be a reason why the impact of a hub on networks and infrastructure planning is a subject that still requires further research.

The representation of other existing connections also would allow the use of different routes between origins and destination. In this case, the resulting graph would also include transit nodes, resembling transshipment models. This is actually the generic formulation for network flow problems, from which simplifications are made to reach HLP models (Campbell \& O'Kelly, 2012; Ragsdale, 2014). Hence, an expanded representation would remain compatible to this class of problems. Yet it should be noted that the simplifications made are closely related to the complexity of solving HLP problems with larger graphs, once this is a NP-hard problem, as 
well as to the availability of models and solving techniques that would enable us to find a viable solution in a timely manner.

There are some applications where the classic HLP model would be well suited. Logistics hubs have been long dedicated to air transportation or postal services (Allaz, 2005). In these cases, transportation via hub is mandatory and direct connections do not make much sense, either with regards to the transportation mode used or to the characteristics of the service performed. The wide adoption of HLP models may also be related to the data sets mostly used to validate the proposed formulations, which are regarded to airport networks (such as the CAB dataset, introduced by O'Kelly in 1986) or postal operations (such as the AP dataset, introduced by Ernst \& Krishnamoorthy in 1996). However, this might lead authors to disregard features that are common to other scenarios.

When locating a logistics hub, it may also be interesting to evaluate the available infrastructure and the need to build new links in order to improve the transportation performance. According to Campbell \& O'Kelly (2012), there exists a direct relationship between location problems and network design. However, the design of large-scale networks with a variety of connections and logistics hubs is still a challenge, especially if we want to include this in the location model. Clearly, a free network design where many new links could be established would add great complexity to the model and be in conflict with the investment capacity of a region. Alumur, Kara \& Karasan (2012) highlight these issues, proposing a framework that considers just a few possibilities of new arcs. In the same direction, sets of new projects could be formulated, simplifying the model to test pre-defined network topologies.

Since neither of the two types of models alone allows us to tackle all matters related to locating a logistics hub, an analytical modelling approach seems to be more suitable, combining features of both multi-criteria and single-criterion models. A multi-criteria model, taking into account strategic matters of hub positioning and regional competitiveness, could be adopted initially to define a location site, or a list of them. The results would then be used as an input for a more generic network flow model; i.e. a transshipment model. With this, a network could be represented in greater detail, allowing for the choice between different routes and assessment of the hubbing effect on the flow's distribution. This network flow model could take into account microeconomic perspectives for decision making, addressing transportation cost reduction and other issues related to the benefits that could obtained by hub users. Thus, we would be able to combine both perspectives in one approach for solving the problem.

Regarding the solutions techniques, we did not find a preference in the literature; nevertheless, we were able to identify some adoption patterns. There is a correlation between the models formulated and the solution techniques adopted: the degree of complexity used to represent the problem defines, in a certain way, the tools implemented. Quantitative methods, for example, are not traditionally the first choice when dealing with strategic location decisions, given the difficulty in obtaining information and processing the available data (Melo, Nickel \& Saldanhada-Gama, 2009). Besides, the fact that MCDM tools are able to handle many, and sometimes conflicting, variables may also explain the preference for this kind of method when solving multi-criteria models. 
The choice of solution methods for single-criterion models, however, seems to be directly related to models' characteristics and the amount of time available to find a solution. A more detailed network and an increase in the volume and variety of product flows add computational challenges, due to the greater number of connections and constraints to be considered. Škrinjar, Rogić \& Stancović (2012) point out that HLP models of small instances can be solved with exact methods, while larger problems require, in general, the use of heuristics. Accordingly, Ambrosino \& Sciomachen (2012) assert that real world problems, usually characterized by a large volume of data, are also generally solved with heuristic tools. This is closely related to the combinatorial nature of these problems. The adoption of heuristics is related to the amount of time available to find a solution: they tend to achieve it in faster computational times. On the other hand, if we want to add uncertainty, stochastic or robust optimization could be good choices of tools.

Meanwhile, some evidence shows that new algorithmic and computational developments have enabled the use of exact methods for solving larger HLP models, with over 500 nodes of origin and destination (Campbell \& O'Kelly, 2012). In this direction, Škrinjar, Rogić \& Stancović (2012) suggest that methods which adopt extensive search could benefit from aggregating Branch-and-Bound and Branch-and-Cut techniques in order to lower computing time by reducing the problems' dimensions.

\section{CONCLUSIONS}

The growing importance of logistics hubs as an element of transportation networks fosters the study and definition of their features, as well as the development of knowledge on how to deal with such structures. To shed light in this area, this paper presented a literature review on logistics hubs location. We surveyed models and solution techniques available, and assessed their applicability within this context. This work differs from others in the field of location science by evaluating an application area instead of a class of models or methods. It facilitates a better understanding of requirements and of how to solve this type of location problem.

We identified two categories of models which are used in logistics hubs location. Multi-criteria models enable the consideration of a broad range of criteria, both quantitative and qualitative, which makes them more suitable for representing such strategic decisions. However, they provide information only about location sites, and do not allow the assessing of the distribution of flows and their impact on the network infrastructure. Single-criterion models, on the other hand, tend be similar to the HLP and deliver results related not only to hub location, but also to flow allocation. Because of these features they might seem, at first glance, more suitable and complete. Yet they adopt network simplifications that do not correspond to a correct representation of the transport system and the connections between origins, hubs, and destinations.

It is also noteworthy that the papers surveyed adopt mainly two different research approaches, which are directly related to the type of models and solution techniques employed. While the multi-criterion category follows an empirical design approach, where the goal is to create models that better represent the existing relationships in real world problems, the single-criterion one 
has an axiomatic perspective, where the primary interest is to understand the modeling process, explain its characteristics, find an optimal solution, and compare the performance of different solution techniques. This contrast of approaches is emphasized by the different perspectives taken by each category: macroeconomic versus microeconomic.

Perhaps a better way to address logistics hub location would be by considering aspects of both categories - a two stage analytical approach through the combination of different features. First, a multi-criteria analysis could be used to define a location site or a ranked list of sites, taking into account political, legal, environmental, and market aspects, among others. Then, the implementation of a network flow model based on economic and/or business criteria would not only aid in defining the allocation of flow, but also allow the evaluation of changes in the use of infrastructure due to the installation of one or more hubs. This would furthermore enable the assessment of issues related to network design, by testing different sets of infrastructure projects and evaluating their impact on an integrated transportation networks considering logistics hubs. Solving tools could be chosen respectively.

There is, indeed, a stated need for a more refined representation of transportation networks. Rahimi, Asef-Vaziri \& Harrison (2008), Ambrosino \& Sciomachen (2012) and Alumur, Kara $\&$ Karasan (2012) are in agreement on the importance of considering different decision criteria, which are relevant and inherent to logistics hubs and their role in transportation networks. In this context, issues related to environmental impact, proximity to transportation modes, traffic, congestion, and volume of flow handled at the hub still require further investigation. On the other hand, Škrinjar, Rogić \& Stancović (2012) point out the importance of studying network topologies where transportation can be done either via hub or by direct connections, which are scarce in the literature. According to Dubke \& Pizzolato (2011), future research should also look at network design and infrastructure planning, comprising a variety of transportation modes such as road-, rail- and waterways. In addition, the impacts of a new hub on the network should be further explored Farahani et al. (2013). Since all of this adds to the complexity of models, the search for new solution algorithms and improvements in computational power also find room in the logistics hub location context.

\section{ACKNOWLEDGMENTS}

This research was supported by $\mathrm{CNPq}$ - National Council for Scientific and Technological Development in Brazil. We thank to the anonymous referees for their valuables comments to improve this review.

\section{REFERENCES}

[1] Allaz C. 2005. History of Air Cargo and Airmail from the 18th Century. London: Christopher Foyle Publishing. 1st ed.

[2] Alumur S \& Kara BY. 2008. Network hub location problems: The state of the art. European Journal of Operational Research, 190(1): 1-21. 
[3] Alumur S, Kara BY \& Karasan OE. 2012. Multimodal hub location and hub network design. Omega, 40(6): 927-939.

[4] Ambrosino D \& Sciomachen A. 2012. Hub locations in urban multimodal networks. European Transport-Trasporti Europei, 51: 1-14.

[5] Boudouin D \& Luna MMM. 2012. Plano Estadual de Logística e Transportes: relatório de atividades de consultoria. Florianópolis.

[6] Campbell JF \& O'Kelly ME. 2012. Twenty-five years of hub location research. Transportation Science, 46(2): 153-169.

[7] Ding JF. 2013. Applying an integrated fuzzy MCDM method to select hub location for global shipping carrier-based logistics service providers. WSEAS Transactions on Information Science and Applications, 10(2): 47-57.

[8] DubKe AF \& Pizzolato ND. 2011. Location model of specialized terminals for soybean exports in Brazil. Pesquisa Operacional, 31(1): 21-40.

[9] ERnst AT \& Krishnamoorthy M. 1996. Efficient algorithms for the uncapacitated single allocationp-hub median problem. Location Science, 4(3): 139-154.

[10] Farahani RZ, Hekmatfar M, Arabani AB \& Nikbakhsh E. 2013. Hub location problems: A review of models, classification, solution techniques, and applications. Computers \& Industrial Engineering, 64(4): 1096-1109.

[11] FARAhani RZ, SeIFI MS \& ASGari N. 2010. Multiple criteria facility location problems: A survey. Applied Mathematical Modelling, 34(7): 1689-1709.

[12] Feng F, Li F \& Zhang Q. 2013. Location selection of Chinese modern railway logistics center based on DEA-Bi-level programming model. Research Journal of Applied Sciences, Engineering and Technology, 6(5): 812-818.

[13] Gao M \& Dong M. 2012. Analysis of Logistics Center Location-Selecting Based on GIS-Take Li County as an Example. Advanced Materials Research, 569: 804-807.

[14] Goldman AJ. 1969. Optimal location for centers in a network. Transportation Science, 3(4): 352360.

[15] KAMPF R, Prů̌̌ P \& SAVAge C. 2011. Systematic location of the public logistic centres in Czech Republic. Transport, 26(4): 425-432.

[16] KARA BY \& TANeR MR. 2011. Foundations of Location Analysis. In Foundations of Location Analysis, 155: 25-38. Springer US.

[17] Kimms A. 2006. Economies of Scale in Hub \& Spoke Network Design Models: We Have It All Wrong. In Perspectives on Operations Research, pp. 293-317. DUV.

[18] Klapita V \& ŠVeCovÁ Z. 2006. Logistics centers location. Transport, 21(1): 48-52.

[19] KLINCEWICZ JG. 1998. Hub location in backbone/tributary network design: a review. Location Science, 6(1-4): 307-35.

[20] LeE KL, HuAng WC \& Teng JY. 2009. Locating the competitive relation of global logistics hub using quantitative SWOT analytical method. Quality and Quantity, 43(1): 87-107.

[21] Li Y, LiU X \& CHEN Y. 2011. Selection of logistics center location using Axiomatic Fuzzy Set and TOPSIS methodology in logistics management. Expert Systems with Applications, 38(6): 7901-7908. 
[22] LiU X, GuO X \& ZHaO X. 2012. Study on logistics center site selection of jilin province. Journal of Software, 7(8): 1799-1806.

[23] Lium A-G, Crainic TG \& Wallace SW. 2009. A Study of Demand Stochasticity in Service Network Design. Transportation Science, 43(2): 144-157.

[24] Melo MT, Nickel S \& Saldanha-DA-Gama F. 2009. Facility location and supply chain management - A review. European Journal of Operational Research, 196(2): 401-412.

[25] O' Kelly ME. 1986. The Location of Interacting Hub Facilities. Transportation Science, 20(2): 92-106.

[26] OKtal H \& Ozger A. 2013. Hub location in air cargo transportation: A case study. Journal of Air Transport Management, 27: 1-4.

[27] Ragsdale C. 2014. Spreadsheet Modeling and Decision Analysis: A Practical Introduction to Business Analytics. Stanford: Cengage Learning. 7th ed.

[28] RAhimi M, ASEF-VAziri A \& HARrison R. 2008. An inland port location-allocation model for a regional intermodal goods novement system. Maritime Economics and Logistics, 10(4): 362-379.

[29] Ren Y-C, XING T, QUAN Q \& ZHaO G-Q. 2010. Fuzzy cluster analysis of regional city multi-level logistics distribution center location plan. Advances in Intelligent and Soft Computing, 82: 499-508.

[30] Simchi-Levi D, Kaminsky P \& Simchi-Levi E. 2003. Managing the Supply Chain: The Definitive Guide for the Business Professional. New York: McGraw Hill Book Co.

[31] ŠKRINJAR JP, RogiĆ K \& STANCOVIĆ R. 2012. Location of urban logistic terminals as hub location problem. Promet - Traffic - Traffico, 24(5): 433-440.

[32] TU C-S, Chen K-K, ChAng C-T \& LU H-A. 2010. Applying an AHP - QFD conceptual model and zero-one goal programming to requirement-based site selection for an airport cargo logistics center. International Journal of Information and Management Sciences, 21(4): 407-430.

[33] TURSKis Z \& ZAVAdskAS EK. 2010. A new fuzzy additive ratio assessment method (ARAS-F). Case study: The analysis of fuzzy Multiple Criteria in order to select the logistic centers location. Transport, 25(4): 423-432.

[34] WANG B-H \& HE S-W. 2009. Robust optimization model and algorithm for logistics center location and allocation under uncertain environment. Jiaotong Yunshu Xitong Gongcheng Yu Xinxi/ Journal of Transportation Systems Engineering and Information Technology, 9(2): 69-74.

[35] XiAO L \& Zhang Q. 2009. A hybrid ant colony algorithm for the grain distribution centers location. In: Emerging Intelligent Computing Technology and Applications - series Lecture Notes in Computer Science, 5754: 112-119.

[36] ZHI G \& Li Z. 2012. An improved ant colony algorithm for the location of logistics center. Applied Mechanics and Materials, 209-211: 887-890.

[37] Zhi J, LiU J-Y, WANG W, WU H-P \& GAO J. 2010. Logistics center location selection based on the algorithm of hybrid particle swarm optimization. Key Engineering Materials, 429-433. 